Acta Crystallographica Section D

\section{Biological Crystallography}

ISSN 0907-4449

\title{
The structures of Arabidopsis Deg5 and Deg8 reveal new insights into HtrA proteases
}

Plant Deg5 and Deg8 are two members of the HtrA proteases, a family of oligomeric serine endopeptidases that are involved in a variety of protein quality-control processes. These two HtrA proteases are located in the thylakoid lumen and participate in high-light stress responses by collaborating with other chloroplast proteins. Deg5 and Deg8 degrade photodamaged D1 protein of the photosystem II reaction centre, allowing its in situ replacement. Here, the crystal structures of Arabidopsis thaliana Deg5 (S266A) and Deg8 (S292A) are reported at 2.6 and $2.0 \AA$ resolution, respectively. The Deg5 trimer contains two calcium ions in a central channel, suggesting a link between photodamage control and calcium ions in chloroplasts. Previous structures of HtrA proteases have indicated that their regulation usually requires C-terminal PDZ domain(s). Deg5 is unique in that it contains no PDZ domain and the trimeric structure of Deg5 (S266A) reveals a novel catalytic triad conformation. A similar triad conformation is observed in the hexameric structure of the single PDZ-domain-containing Deg8 (S292A). These findings suggest a novel activation mechanism for plant HtrA proteases and provide structural clues to their function in light-stress response. wgong@sun5.ibp.ac.cn work.

Correspondence e-mail: liulin@ibcas.ac.cn,
₹ These authors contributed equally to this wang

\author{
Laboratory of Non-coding RNA, Institute of \\ ${ }^{\mathbf{b}}$ Photosynthesis Research Center, Key \\ Cotobiology, Institute of Botan \\ Academy of Sciences, 19A Yuquan Road, \\ Beijing 100049, People's Republic of China \\ Wei Sun, ${ }^{\mathrm{a}, \mathrm{c}} \neq$ Feng Gao, ${ }^{\mathrm{a}} \neq$ \\ Haitian Fan, ${ }^{\mathrm{a}, \mathrm{c}} \mp$ Xiaoyue Shan, ${ }^{\mathrm{a}, \mathrm{c}}$ \\ Renhua Sun, ${ }^{b, c}$ Lin Liu ${ }^{b} *$ and
}

Received 18 December 2012 Accepted 21 January 2013

PDB References: Deg5, 4ic5; Deg8, 4ic6

\section{Introduction}

HtrA (high-temperature requirement A) proteases are a family of ATP-independent trypsin-like serine endopeptidases that belong to MEROPS subfamily S1C and are involved in protein quality control (PQC) in both prokaryotes and eukaryotes (Rawlings et al., 2008; Clausen et al., 2002; Ehrmann \& Clausen, 2004). They have an N-terminal protease domain and 0-3 PDZ (PSD95/Dlg1/ZO-1) domain(s) at the $\mathrm{C}$-terminal end. The functional unit of the HtrA proteases is a trimer, with three protease domains forming a central core. The physiological importance of the HtrA proteases can be inferred from their wide distribution from bacteria to humans. Three HtrA proteases from Escherichia coli, DegS, DegP and DegQ, are the best-characterized prokaryotic members (Singh et al., 2011). They are located in the periplasmic space and are involved in essential PQC processes. Human HtrA1 and HtrA2 are the most thoroughly studied eukaryotic members and both have been discovered to play key roles in development and disease (Clausen et al., 2011).

Their lack of motility renders plants constantly susceptible to environmental stresses. To deal with photodamage to their photosynthetic machinery, plants have evolved a sophisticated 
Table 1

Data-collection and refinement statistics for Deg5 (S266A) and Deg8 (S292A)

\begin{tabular}{|c|c|c|}
\hline & Deg5 (S266A) & Deg8 (S292A) \\
\hline \multicolumn{3}{|l|}{ Data collection } \\
\hline Space group & $C 2$ & $C 2$ \\
\hline Unit-cell parameters $\left(\AA{ }^{\circ},{ }^{\circ}\right)$ & $\begin{array}{r}a=109.1, b=126.0, \\
\quad c=83.3, \beta=102.9\end{array}$ & $\begin{array}{r}a=129.5, b=124.2, \\
c=93.3, \beta=132.4\end{array}$ \\
\hline Resolution range $(\AA)$ & $50-2.6(2.69-2.60)$ & $50-2.0(2.07-2.00)$ \\
\hline $\begin{array}{l}\text { No. of reflections } \\
\text { (total/unique) }\end{array}$ & $33072 / 11024$ & $73478 / 9929$ \\
\hline$\langle I / \sigma(I)\rangle$ & $10.9(2.3)$ & $25.2(4.3)$ \\
\hline Completeness (\%) & $99.2(97.8)$ & $99.3(100.0)$ \\
\hline Multiplicity (\%) & $3.0(2.9)$ & $7.4(7.7)$ \\
\hline Wilson $B$ factor $\left(\AA^{2}\right)$ & 50.2 & 37.5 \\
\hline$R_{\text {merge }}(\%)$ & $9.4(46.5)$ & $6.7(47.8)$ \\
\hline \multicolumn{3}{|l|}{ Structure refinement } \\
\hline Resolution $(\AA)$ & $80-2.6$ & $30-2.0$ \\
\hline$R_{\text {work }}$ & 0.182 & 0.198 \\
\hline$R_{\text {free }}$ & 0.231 & 0.235 \\
\hline \multicolumn{3}{|l|}{ Ramachandran plot (\%) } \\
\hline Favoured region & 93.1 & 96.4 \\
\hline Allowed region & 6.1 & 3.4 \\
\hline Outlier region & 0.8 & 0.2 \\
\hline R.m.s.d. bonds $(\AA)$ & 0.007 & 0.007 \\
\hline R.m.s.d. angles $\left({ }^{\circ}\right)$ & 1.026 & 1.122 \\
\hline Mean $B$ value $\left(\AA^{2}\right)$ & 51.7 & 51.8 \\
\hline
\end{tabular}

PQC system (Sakamoto, 2006; Eberhard et al., 2008). The members of the HtrA proteases have been identified as critical components of the chloroplast PQC system (Schuhmann et al., 2012; Komenda et al., 2012). Among the 16 Arabidopsis HtrA proteases that have been annotated, Deg1, Deg5 and Deg8 are in the chloroplast thylakoid lumen (Schuhmann \& Adamska, 2012). Deg1 has been reported to be involved in photoinhibition repair and photosystem II (PSII) assembly through its dual protease and chaperone activity (Kapri-Pardes et al., 2007; Sun, Ouyang et al., 2010). Deg5 and Deg8 are required for efficient PSII repair under light stress and recombinant Deg8 is proteolytically active with $\beta$-casein as the substrate, whereas Deg5 demonstrates no $\beta$-casein degradation activity (Sun et al., 2007; Kato et al., 2012). Deg1, Deg5 and Deg8 together with two stromal HtrA members, Deg2 and Deg7 (Haussühl et al., 2001; Sun, Fu et al., 2010), participate in degradation of damaged reaction-centre protein D1 in the PSII complex during light stress.

The structures of members of the HtrA proteases from E. coli (DegS, DegP and DegQ) and of human HtrA1 and HtrA2 have provided detailed insights into their activation mechanisms. Trimeric DegS requires the binding of stresssignalling peptides to its PDZ domain for allosteric activation (Wilken et al., 2004; Sohn et al., 2007), while DegP and DegQ form large oligomers with encapsulated substrate (Krojer et al., 2008; Kim et al., 2011; Malet et al., 2012). Unlike DegS, the HtrA1 trimer and the HtrA2 trimer can degrade substrates directly without any other cofactors (Truebestein et al., 2011; Li et al., 2002).

Recent structural studies on Arabidopsis Deg1 have suggested a pH-dependent activation pathway (Kley et al., 2011). It has been shown that loops LA, L1, L2, L3 and LD play direct roles in the regulation of Deg1 activity. At acidic $\mathrm{pH}$, Deg1 trimers dimerize through interactions between the
PDZ domain and loop LA of the protease domain, leading to a fixed position of the PDZ domain and establishing a PDZL3-LD interaction network. As it is naturally devoid of a PDZ domain, Deg5 should employ a different activation mechanism. Although Deg8 is the closest homologue of Deg1 in the Arabidopsis genome (Schuhmann \& Adamska, 2012), these two enzymes are functionally different. Deg1 is constitutively expressed and is required for plant growth, while Deg8 is sensitive to high light levels and together with Deg5 does not contribute significantly to the degradation of PSII D1 under low-light conditions (Kato et al., 2012). In addition, Deg5 and Deg8 have a synergistic effect on PSII D1 degradation during high light stress (Sun et al., 2007). All of these facts indicate that Deg5 and Deg8 utilize different mechanisms to regulate protease activity and the structural basis behind them needs to be examined.

Here, we report the crystal structures of Deg5 (S266A) and Deg8 $(\mathrm{S} 292 \mathrm{~A})$ at 2.6 and $2.0 \AA$ resolution, respectively. Both structures represent proteolytically incompetent states. The catalytic triad in both structures is malformed by rotation of the conserved histidine. We also find that the Deg5 trimer can bind two calcium ions. These findings provide novel structural evidence of the differing activities and regulation mechanisms of HtrA proteases.

\section{Materials and methods}

\subsection{Expression, purification and crystallization}

The expression, purification, crystallization and preliminary diffraction analysis of Deg5 (S266A) and Deg8 (S292A) have been described previously (Fan et al., 2012; Shan et al., 2013).

\subsection{Data collection and structure determination}

All data collections were performed on beamline BL17U at Shanghai Synchrotron Radiation Facility (SSRF) using a MAR 225 CCD detector (MAR Research). Data were processed with $H K L-2000$ (Otwinowski \& Minor, 1997).

Deg5 crystallized in space group $C 2$, with three molecules per asymmetric unit that are related by a noncrystallographic symmetry threefold axis. The structure of Deg5 (S266A) was solved by the molecular-replacement method using MOLREP (Vagin \& Teplyakov, 2010) within the CCP4 suite (Winn et al., 2011). HtrA from Thermotoga maritima (PDB entry 111j; Kim et al., 2003) was used as the search model. The initial model was built with $A R P / w A R P$ (Cohen et al., 2008) and amino acids were then added manually in Coot (Emsley et al., 2010). Refinement was performed with REFMAC5 (Murshudov et al., 2011). Noncrystallography symmetry restraints were used. The final $R$ and $R_{\text {free }}$ were $18.2 \%$ and $23.1 \%$, respectively.

Deg8 (S292A) crystals were soaked in cryoprotectant $(12 \%$ 2-methyl-2,4-pentanediol) and data collection was performed at a wavelength of $0.9792 \AA$ in a nitrogen stream at $100 \mathrm{~K}$. The Deg8 crystal belonged to space group $C 2$ and one asymmetric unit contained three Deg8 molecules, which are also related by a noncrystallographic symmetry threefold axis. The Deg8 (S292A) structure was also solved by molecular replacement 


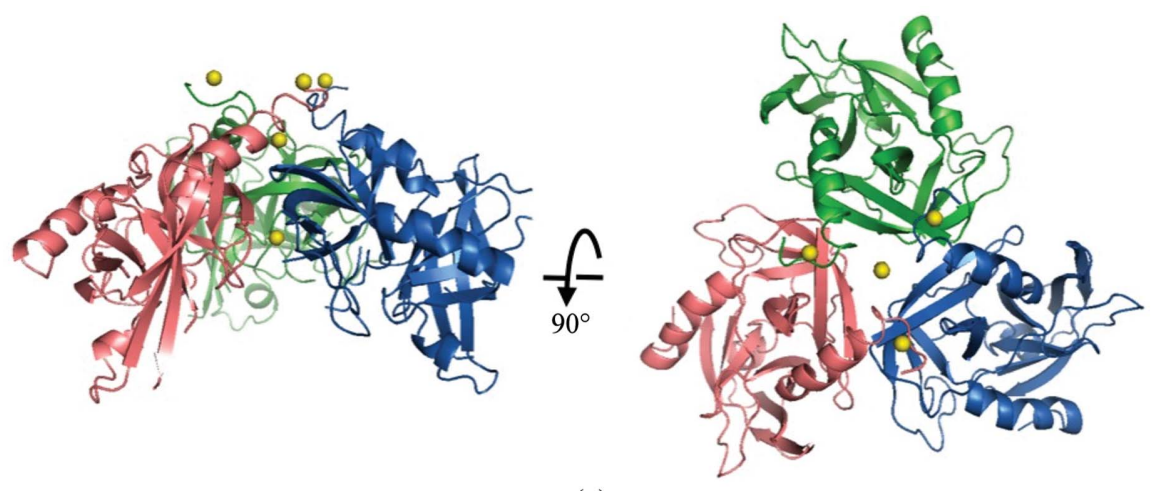

(a)
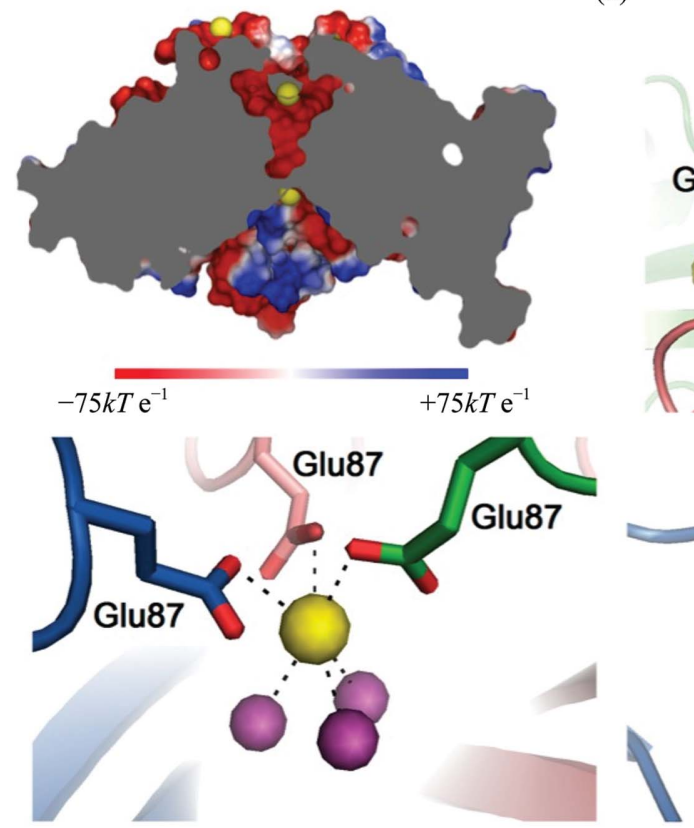

(b)

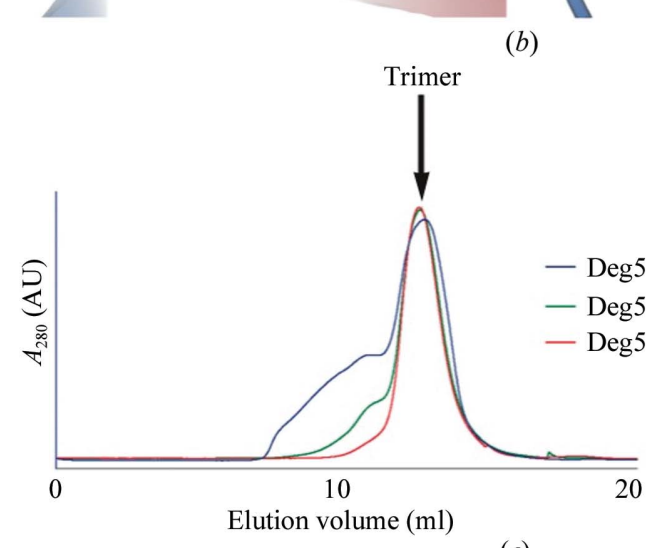

(c)

Asp260

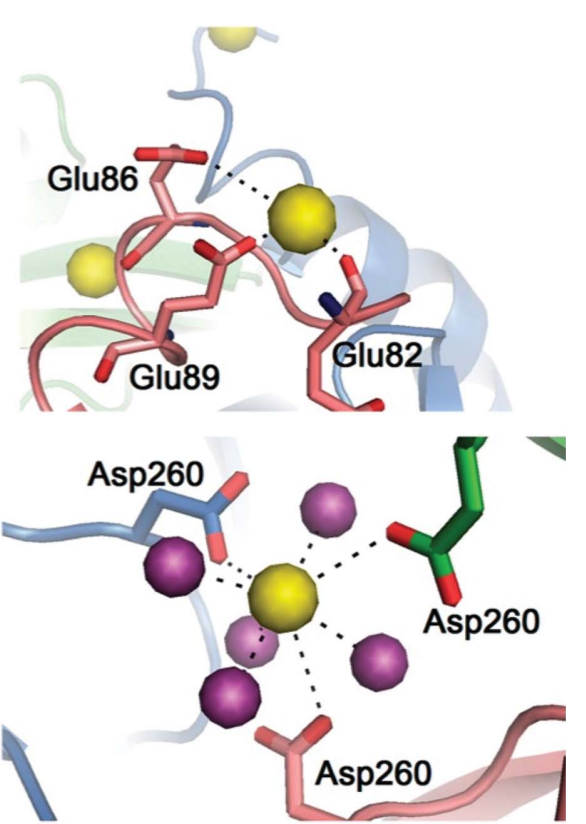

Figure 1

Overall structure of Deg5 (S266A) and binding of $\mathrm{Ca}^{2+}$ ions. (a) Side and top views of the Deg5 (S266A) trimer with each protomer coloured differently. (b) $\mathrm{Ca}^{2+}$ ions binding to Deg5 (S266A). Top and left, electrostatic potential distributions of the inner surface of the trimer. Electrostatic potential was calculated using PyMOL. Blue and red represent positive and negative charge potentials on a scale between +75 and $-75 k T \mathrm{e}^{-1}$, respectively. Top and right, $\mathrm{Ca}^{2+}$ ions binding to the convex side of the trimer. Bottom, $\mathrm{Ca}^{2+}$ ions binding to the central channel of the Deg5 trimer. $\mathrm{Ca}^{2+}$ ions and waters are shown as spheres and are coloured yellow and purple, respectively. Residues interacting with $\mathrm{Ca}^{2+}$ ions are shown as sticks and are labelled. Salt bridges formed between Deg5 and $\mathrm{Ca}^{2+}$ ions are presented as black dashed lines. (c) Analytical SEC of Deg5 (S266A) (blue), Deg5 (S266A) dialyzed against EDTA (red) and Deg5 (S266A/E87A/D260A) triple mutant (green). The trimeric peak is indicated by an arrow. and the search model for Deg8 (S292A) was the structure of Arabidopsis Deg1 (PDB entry 3qo6; Kley et al., 2011), including both the protease and PDZ domains. The model was refined with PHENIX (Adams et al., 2010) and CNS (Brünger et al., 1998) and was built using Coot (Emsley et al., 2010) based on the $F_{\mathrm{o}}-F_{\mathrm{c}}$ electron-density map. Further refinement was performed with REFMAC5 (Murshudov et al., 2011). The final $R$ and $R_{\text {free }}$ were $19.8 \%$ and $23.5 \%$, respectively.

The final models of Deg5 (S266A) and Deg8 (S292A) were validated with PROCHECK (Laskowski et al., 1993). Statistics of data collection and refinement are summarized in Table 1.

\subsection{Inductively coupled plasma mass spectrometry (ICP-MS)}

Analysis of calcium content was carried out using ICP-MS (Thermo Scientific) at the Tsinghua University Analysis Center (Beijing, People's Republic of China). Purified Deg5 (S266A) was dialyzed thoroughly against buffer consisting of $20 \mathrm{~m} M$ Tris$\mathrm{HCl}$ pH 8.0, $150 \mathrm{~m} M \mathrm{NaCl}$ before ICPMS. To obtain calcium-stripped samples, $5 \mathrm{~m} M$ EDTA was added to the dialysis buffer. The ICP-MS data are summarized in Table 2.

\subsection{Oligomeric state analysis}

$10 \mu M$ protein was buffered in sodium phosphate $\mathrm{pH} 6.0$ or 8.0, $150 \mathrm{~m} M \mathrm{NaCl}$ and was incubated on ice for at least $1 \mathrm{~h}$. Samples were then loaded onto a Superdex 200 10/300 GL (GE Healthcare) size-exclusion column and their molecular weights were calculated using a calibration curve determined using calibration standards (GE Healthcare).

\subsection{Proteolytic activity assay}

For each assay, $100 \mu \mathrm{g} \beta$-casein was incubated in $200 \mu \mathrm{l} 50 \mathrm{mM}$ sodium phosphate $\mathrm{pH} 6.0,50 \mathrm{~m} M \mathrm{NaCl}$ with or without $20 \mu \mathrm{g}$ recombinant Deg8 or $2 \mu \mathrm{g}$ recombinant Deg1 at $310 \mathrm{~K}$. The reaction mixtures at different time points were subjected to SDS-PAGE on a $12 \%$ acrylamide gel. 


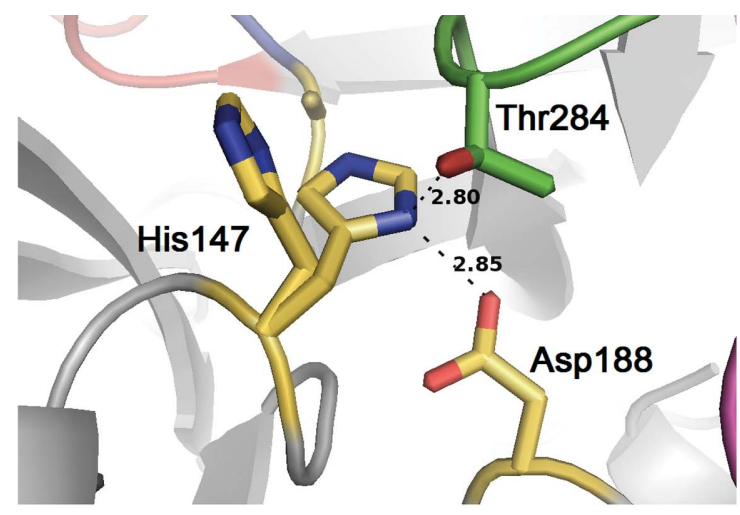

(a)
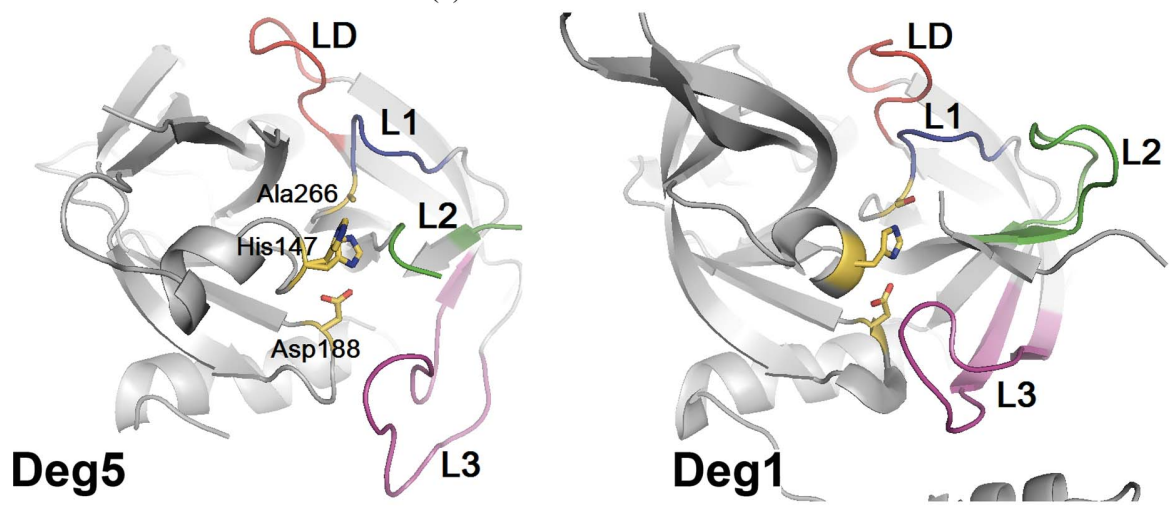

Deg1

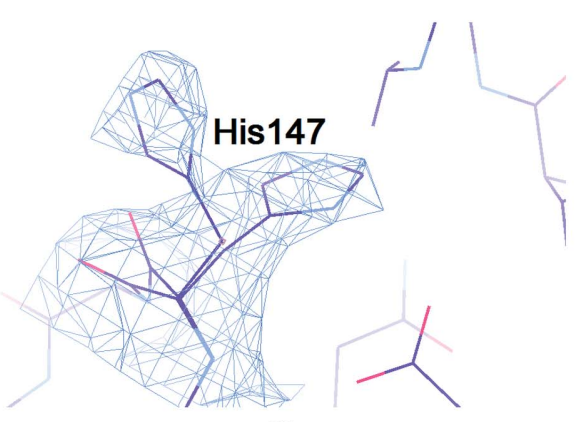

(b)

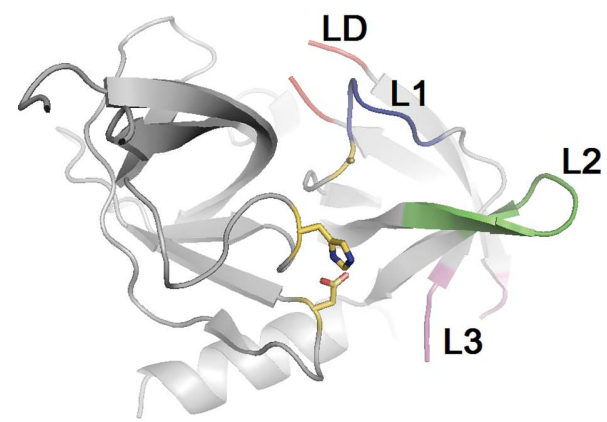

HtrA1

Figure 2

Structural comparison of the active sites of Deg5 (S266A), Deg1 and HtrA1. (a) Catalytic triad of Deg5 (S266A) (yellow). Hydrogen bonds between His147 and Asp188 and between His147 and Thr284 are presented as black dashed lines. The catalytic triad and Thr284 from loop L2 are shown in stick mode. (b) Alternative conformation of His147. His147 is displayed together with electron density calculated using $2 F_{\mathrm{o}}-F_{\mathrm{c}}$ coefficients and contoured at $0.7 \sigma$ as a blue mesh. (c) Structural comparison of catalytic triads and activation loops of Deg5 (S266A), Deg1 (PDB entry 3qo6) and HtrA1 (PDB entry 3num; Truebestein et al., 2011). Catalytic triads are shown in stick mode and are coloured yellow. Loops LD, L1, L2 and L3 are coloured red, blue, green and magenta, respectively.

Table 2

ICP-MS analysis of Deg5 with or without EDTA treatment.

\begin{tabular}{lcll}
\hline Protein sample & $\begin{array}{l}\mathrm{Ca}^{2+} \\
\left(\mathrm{ng} \mathrm{ml}^{-1}\right)\end{array}$ & $\begin{array}{l}\mathrm{Deg} 5 \\
\left(\mathrm{ng} \mathrm{ml}^{-1}\right)\end{array}$ & $\begin{array}{l}{\left[\mathrm{Ca}^{2+}\right]} \\
{[\mathrm{Deg} 5]}\end{array}$ \\
\hline Deg5 (S266A) & 1553 & 2.00 & 0.60 \\
Deg5 (E87A/D260A/S266A) & 316 & 2.00 & 0.12 \\
Deg5 (S266A) with EDTA treatment & 85 & 1.76 & 0.037 \\
\hline
\end{tabular}

\section{Results and discussion}

\subsection{Structure of $A$. thaliana Deg5 (S266A)}

His147, Asp188 and Ser266 constitute the catalytic triad of Deg5. To avoid self-degradation of wild-type Deg5 during crystallization, Ser266 was mutated to alanine (Fan et al., 2012). The crystal of Deg5 (S266A) belonged to space group $C 2$ and contains a trimer with a threefold noncrystallographic axis of symmetry in the asymmetric unit (Fig. 1a). The trimer has a shallow funnel shape and the catalytic triad is located on the concave side of the funnel. Five $\mathrm{Ca}^{2+}$ ions are observed per trimer. Three are on the convex side and each interacts with the N-terminal acidic residues of each protomer. The other two are located on the noncrystallographic threefold axis, along which a channel is formed (Figs. $1 a$ and $1 b$ ). In the crystal two Deg5 (S266A) trimers associate with each other through their convex surfaces. In the back-to-back hexamer the interactions are mainly hydrogen bonds and salt bridges involving the $\mathrm{Ca}^{2+}$ ions on the surfaces. The molecular weight of Deg5 (S266A) as measured by size-exclusion chromatography (SEC) and dynamic light scattering is approximately $100 \mathrm{kDa}$, indicating that the trimer is the solution species and that two trimers only associate in the crystal.

Trimerization of the protomers is mediated through hydrophobic interactions, hydrogen bonds and a $\pi$-cation interaction. A hydrophobic interface is formed by residues of two regions. One includes Leu231 and Ile233 on $\beta 9$, Phe95 on the N-terminal helix $\alpha 1$, Val235* on $\beta 9$ (where the asterisk denotes the participation of the neighbouring protomer) and Val214* on the loop before $\beta 8$; the other includes Tyr225 on loop LD and Val296* and Phe298* on $\beta 13$ (Supplementary Fig. S $\left.1 a^{\mathbf{1}}\right)$. Hydrogen-bond interactions are found in the area around the hydrophobic interface and include the following

\footnotetext{
${ }^{1}$ Supplementary material has been deposited in the IUCr electronic archive (Reference: DW5040). Services for accessing this material are described at the back of the journal.
} 
pairs: Glu83-Tyr274*, Glu85-Arg90*, Glu88-Ser217*, Glu88Ser273*, Asn91-Gly215*, Gln96-Arg213*, Leu231-Ser237* and Ile233-Asp258* (Supplementary Fig. S1b). Moreover, Tyr227 donates $\pi$-electrons to the $\mathrm{H}$ atom on the $\mathrm{N}^{\varepsilon}$ atom of Arg241*, which also contributes to trimer stabilization (Supplementary Fig. S1c).

\section{2. $\mathrm{Ca}^{2+}$ ions in the central channel of the Deg5 trimer}

The electrostatic surface of the central channel in the Deg5 (S266A) trimer is highly acidic (Fig. 1b, top and left). The carboxyl groups of Glu87 and Asp260 stabilize two $\mathrm{Ca}^{2+}$ ions at both ends of the channel (Fig. 1b, bottom). ICP-MS analysis of the Deg5 (S266A) solution sample quantified that the $\mathrm{Ca}^{2+}$ :protomer ratio was 0.60 , corresponding to $1.8 \mathrm{Ca}^{2+}$ ions per trimer (Table 2). In the crystal there are five $\mathrm{Ca}^{2+}$ ions. The three excess $\mathrm{Ca}^{2+}$ ions bound to the $\mathrm{N}$-terminal acidic residues of Deg5 (S266A) (Fig. 1b, top and right) were probably introduced during crystallization. To test whether the central two $\mathrm{Ca}^{2+}$ ions coordinated by acidic residues (Glu87 and Asp260) are required for Deg5 trimerization, we mutated both Glu87 and Asp260 to alanine. SEC revealed that the mutant
(E87A/D260A/S266A) exists predominantly as a trimer (Fig. 1c). ICP-MS analysis of this triple mutant quantified that the $\mathrm{Ca}^{2+}$ :protomer ratio was 0.12 , corresponding to $0.36 \mathrm{Ca}^{2+}$ ions per trimer. These results suggested that the two $\mathrm{Ca}^{2+}$ ions observed in the crystal structure are also accommodated by the two acidic residues in solution.

Deg5 may act as $\mathrm{Ca}^{2+}$-storage protein in chloroplasts. To test this possibility, Deg5 (S266A) was dialyzed against $5 \mathrm{~m} M$ EDTA and the $\mathrm{Ca}^{2+}$ :protomer ratio was analyzed by ICP-MS (Table 2). The ratio decreased to $0.037 \mathrm{Ca}^{2+}$ ions per protomer and analysis of the oligomeric state using SEC confirmed that Deg5 remained as a trimer (Fig. 1c). These results indicated that the $\mathrm{Ca}^{2+}$ ions can be readily stripped by EDTA and are dispensable for Deg5 trimerization, indicating a potential role of Deg5 in calcium signalling. Calcium signals are critical for plant development and stress adaptation (Kudla et al., 2010). A recent publication proposed that the thylakoid luminal calcium concentration is a signalling mechanism for PSII oxygen-evolving complex assembly and D1 repair (Lohmiller et al., 2012). The Deg5 (S266A) structure reported here thus provides new clues to the function of Deg5 in PSII quality control. Given the importance of chloroplasts
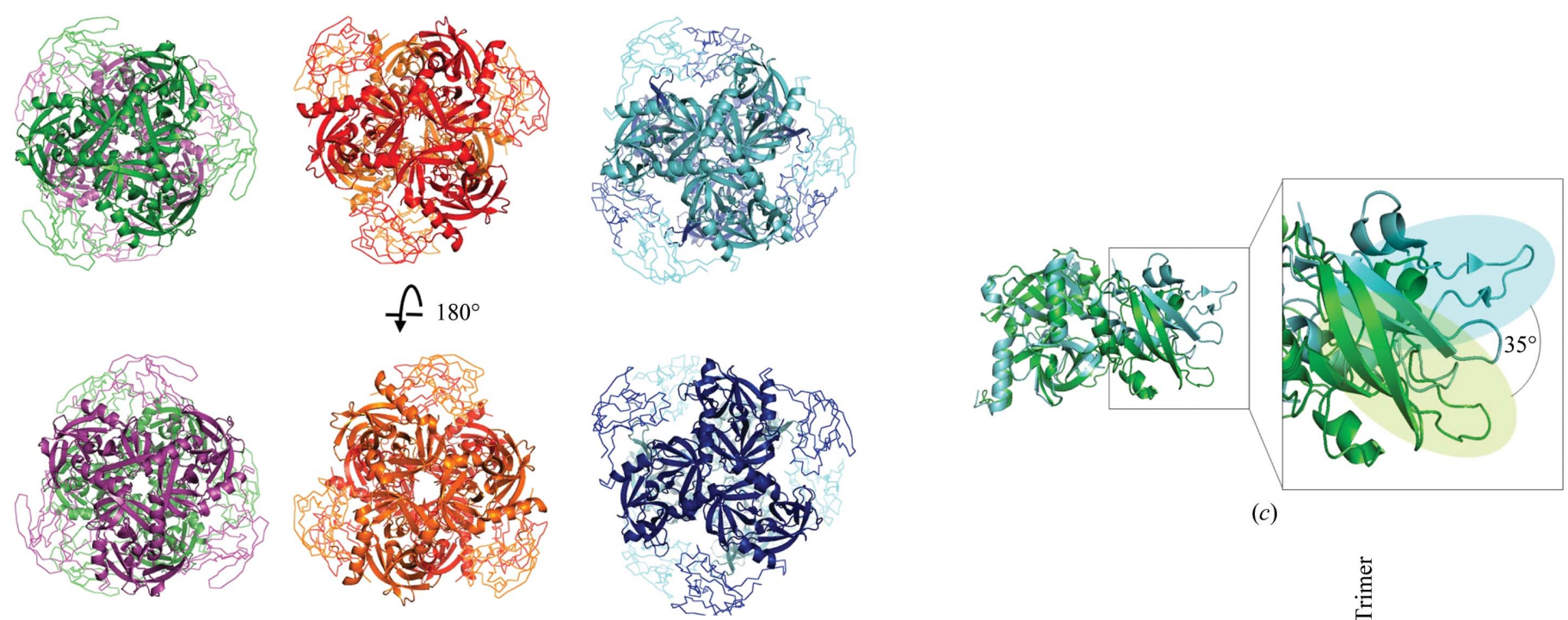

(c)

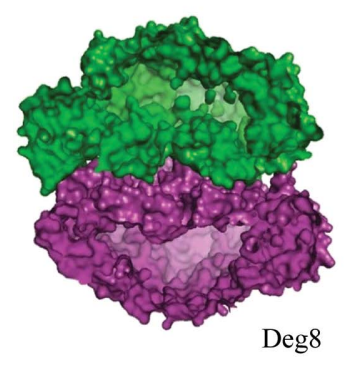

(a)
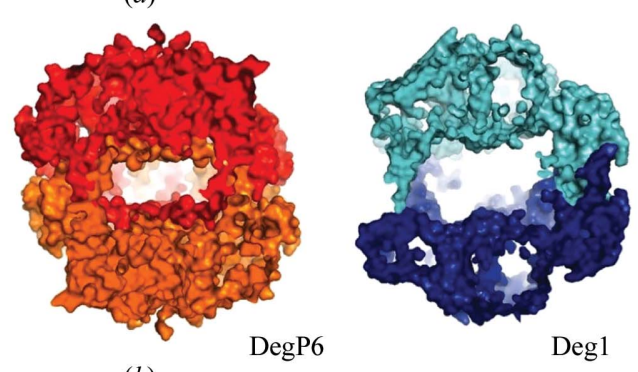

(b)

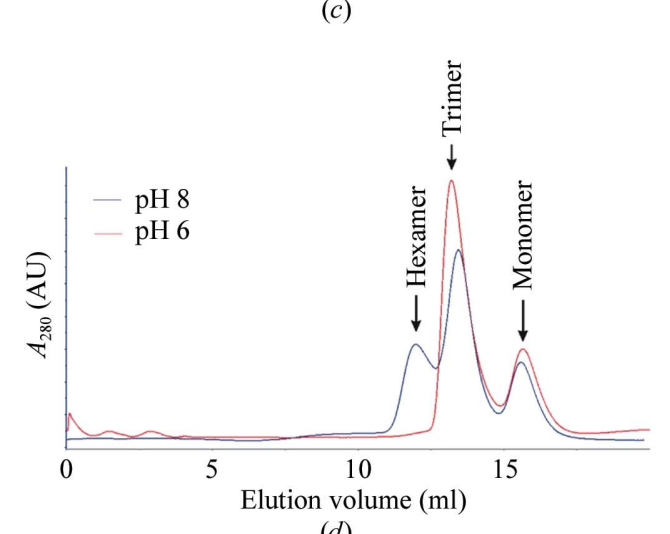

Figure 3

Structure of Deg8 (S292A) in comparison with DegP6 and Deg1 and oligomeric state analysis. (a) Structures of Deg8 (S292A) (green and magenta), DegP6 (PDB entry 1ky9; red and orange; the second PDZ domain is not shown for clarity; Krojer et al., 2008) and Deg1 (PDB entry 3qo6; cyan and blue). Protease domains and PDZ domains are shown in cartoon and ribbon modes, respectively. $(b)$ The cavity formed in Deg8, DegP6 (PDB entry 1ky9; molecule $B$ ) and Deg1 (PDB entry 3qo6). (c) Structural comparison of protomers of Deg8 (S292A) (green) and Deg1 (PDB entry 3qo6; cyan); the difference in PDZ-domain orientations is indicated. $(d)$ Analytical SEC of Deg8 at pH 6.0 (red line) and pH 8.0 (blue line). Different oligomeric states are labelled above the peaks. 
in calcium signalling (Stael et al., 2012), the biological function of $\mathrm{Ca}^{2+}$ ions in the Deg5 central channel awaits further studies.

\subsection{Deg5 active site}

In the Deg5 (S266A) trimer, the side chain of the catalytic His147 has two conformations (Figs. $2 a$ and $2 b$ ). One contains the conserved hydrogen bond between His147 and Asp188, while the alternative conformation differs from that in any reported HtrA structure (Clausen et al., 2011). The $\chi_{1}$ angle of His147 in the alternative conformation is rotated anticlockwise by $\sim 120^{\circ}$, preventing formation of the His147Asp188 hydrogen bond, which is an unusual conformation compared with other distorted HtrA catalytic triads (Krojer et al., 2002; Kim et al., 2003; Wrase et al., 2011). It resembles the catalytic histidine of human HtrA1 (Truebestein et al., 2011; Eigenbrot et al., 2012), but in that case the imidazole ring is rotated clockwise around the $\chi_{1}$ axis (Fig. $2 c$ ).

In Deg5 (S266A), residues 111-125 from loop LA, 155-159 from loop LB and 287-295 from loop L2 cannot be traced in the electron-density map. These loops, together with loop L3 (241-251), which has significantly high $B$-factor values, are flexible in the crystal. Loops L1, L2 and LD are defined as the activation domain, the proper conformation of which indicates activated HtrA proteases (Krojer et al., 2002, 2010; Kley et al., 2011), with L1 being linked to oxyanion-hole formation, L2 to the substrate-specificity pocket and LD being defined as the activation loop. Thr284 of loop L2 stretches close to the active site and forms a hydrogen bond to His147 (Fig. 2a) which blocks substrate from approaching the catalytic triad. The missing electron density for loops including L2, together with the distorted His147, indicates that the Deg5 structure shown here is of an inactive form. Deg5 may become proteolytically active after the conformation of loops L1 and L2 is changed by allosteric or substrate activation.

\subsection{Structure of $A$. thaliana Deg8 (S292A)}

The crystallized Deg8 (S292A) contains 358 amino-acid residues (Leu91-Ser448), of which residues 104-448 could be defined in the electron-density map. Deg8 (S292A) forms a hexamer in the crystals (Figs. $3 a$ and $3 b$ ). Interestingly, when superimposing one trimer on Deg1 or DegP6 (DegP hexamer), the orientation of the opposite trimer of the Deg8 protease domains differs from that of Deg1 but is more similar to that of DegP6 (Fig. 3a). Despite the higher similarity between Deg1 and Deg8, when compared with Deg1 (Kley et al., 2011) the orientation of the Deg8 PDZ domain changes by $\sim 35^{\circ}$ along the major inertia axis (Fig. $3 c$ ). As such, the Deg8 PDZ domain is flattened out from the trimeric core made by the protease domain and the concavity of the trimeric funnel is shallower than in Deg1. The internal cavity of Deg8 (S292A) is smaller than that of the inactive DegP hexamer (Krojer et al., 2002; Fig. 3b), with their volumes being 46143 and $69527 \AA^{3}$, respectively, as calculated by $3 V$ (Voss \& Gerstein, 2010). Such
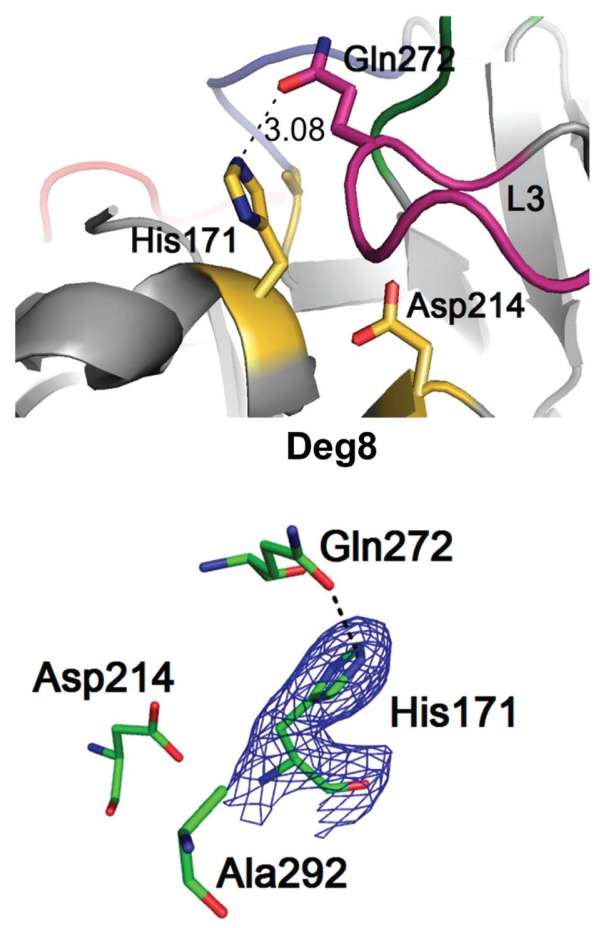

(b)

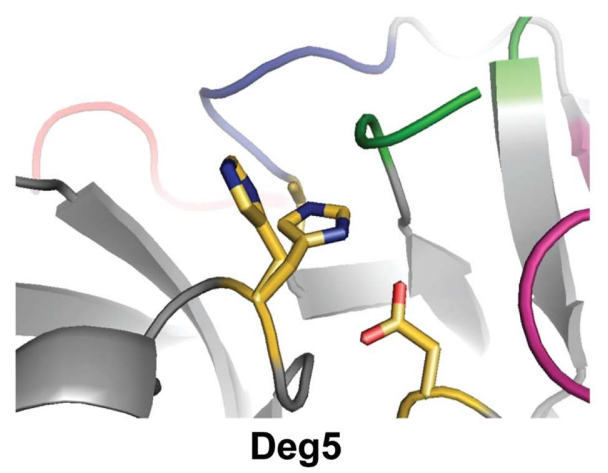

(a)

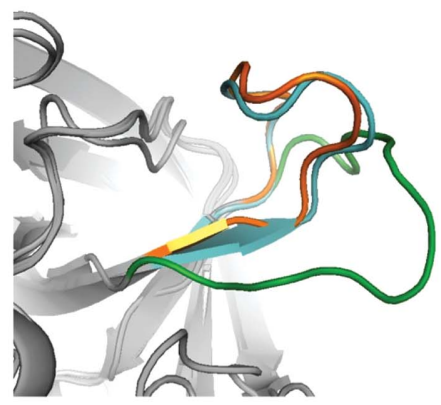

Figure 4

Activation loops and active sites in Deg8 (S292A) and other HtrA proteases. (a) Catalytic triad of Deg8 (S292A) (yellow, stick mode) in comparison with Deg5 (S266A) and HtrA1. The hydrogen bond between His171 and Gln272 from loop L3 is presented as a black dashed line. Activation loops are coloured the same as in Fig. 2(c). (b) Catalytic triad of Deg8 (S292A). His171 is displayed together with electron density calculated using $2 F_{\mathrm{o}}-F_{\mathrm{c}}$ coefficients and contoured at $1.0 \sigma$ as a blue mesh. (c) Structural comparison of loop L2 in Deg8 (S292A) (green), Deg1 (PDB entry 3qo6; cyan; left), DegP24 (PDB entry 3cso; orange; left), Deg5 (blue; right) and HtrA1 (PDB entry 3num; magenta; right). 
a compact hexameric assembly would prevent the substrate from accessing the active site.

Trimerization is primarily mediated by hydrophobic interactions and hydrogen bonds. The residues involved in hydrophobic interactions are Val240, Val261, Phe123*, Leu257*, Pro355, Phe251* and Phe253* (Supplementary Fig. S2a). Seven pairs of hydrogen bonds are found between two neighbouring protomers: Val232-Phe107*, Ser299-Glu116*, Gln243-Glu116*, Asp284-Val259*, Ser263-Leu257*, Ala318Asn288* and Arg267-Asp254* (Supplementary Fig. S2b). Six pairs of hydrogen bonds are observed between Arg179 and Phe389* from opposite trimers, stabilizing the hexamer (Supplementary Fig. S2c). To test the oligomeric state of Deg8 in solution, we performed an analytical SEC assay (Fig. $3 d$ ). The SEC assay showed that Deg8 exists as trimers and monomers at $\mathrm{pH}$ 6.0. However, a small fraction of hexamer appeared at $\mathrm{pH} 8.0$, indicating that $\mathrm{Deg} 8$ tends to form higher oligomers at basic $\mathrm{pH}$ rather than acidic $\mathrm{pH}$ (Fig. $3 d$ ). This behaviour differs from that of Deg1, the hexameric form of which is assembled at low pH (Kley et al., 2011), and from that of Deg2, the hexameric form of which is $\mathrm{pH}$-independent (Sun et al., 2012).

\subsection{Deg8 active site}

The catalytic triad of Deg8 consists of His171, Asp214 and Ser292. In the Deg8 (S292A) structure, however, the triad fails to form catalytically competent hydrogen bonds owing to the anticlockwise rotation of the $\chi_{1}$ angle of His171 by $\sim 120^{\circ}$ (Figs. $4 a$ and $4 b$ ), which is similar to that of Deg5 (S266A) and differs from that of HtrA1 (Truebestein et al., 2011; Eigenbrot et al., 2012; Fig. 4a). His171 can form a hydrogen bond to Gln272 of loop L3, a unique feature that was not previously observed in HtrA proteases (Figs. $4 a$ and $4 b$ ). Moreover, the orientation of loop L2 is quite different from those of other HtrA proteases such as Deg1 (Kley et al., 2011), the DegP 24-mer (Krojer et al., 2008), Deg5 and HtrA1 (Truebestein et al., 2011; Eigenbrot et al., 2012; Fig. 4c). Furthermore, we performed an activity assay on Deg8 using casein as the substrate. The recombinant Deg8 exhibits a remarkably lower proteolytic activity than that of Deg1 in degrading substrate (Supplementary Fig. S2d). This incompetent state suggests that to activate Deg8 a new activation mechanism may be employed for rearrangement of the activation domain.

\section{Conclusions and outlook}

Deg5 is a distinct HtrA protease that is naturally devoid of a PDZ domain. Protease activity has not been detected either in vivo (Sun et al., 2007) or in vitro. As revealed by the Deg5 (S266A) structure and ICP-MS analysis, trimeric Deg5 can bind two $\mathrm{Ca}^{2+}$ ions, suggesting a role in calcium events in chloroplasts. Deg5 (S266A) shows an inactive state in which a novel conformation of the catalytic His147 was observed and Thr284 on loop L2 interacts with His147 and blocks substrate entry. Deg8 contains one PDZ domain that shows a different orientation compared with that in the Deg1 hexamer. The
Deg8 (S292A) hexamer has a narrower cavity and a distorted His171 like His147 in Deg5. Our observation of dramatically different conformations of the catalytic histidine reveals motion of this $\chi_{1}$ angle and provides new structural insights into the regulation mechanism of chloroplast HtrA proteases.

We thank the Shanghai Synchrotron Radiation Facility beamline scientists for technical support during data collection. This work was supported by the Ministry of Science and Technology of China (grants 2011CB910503, 2011CBA00901 and 2012CB917302) and the National Natural Science Foundation of China (grants 31021062 and 31170688).

\section{References}

Adams, P. D. et al. (2010). Acta Cryst. D66, 213-221.

Brünger, A. T., Adams, P. D., Clore, G. M., DeLano, W. L., Gros, P., Grosse-Kunstleve, R. W., Jiang, J.-S., Kuszewski, J., Nilges, M., Pannu, N. S., Read, R. J., Rice, L. M., Simonson, T. \& Warren, G. L. (1998). Acta Cryst. D54, 905-921.

Clausen, T., Kaiser, M., Huber, R. \& Ehrmann, M. (2011). Nature Rev. Mol. Cell Biol. 12, 152-162.

Clausen, T., Southan, C. \& Ehrmann, M. (2002). Mol. Cell, 10, 443-455.

Cohen, S. X., Ben Jelloul, M., Long, F., Vagin, A., Knipscheer, P., Lebbink, J., Sixma, T. K., Lamzin, V. S., Murshudov, G. N. \& Perrakis, A. (2008). Acta Cryst. D64, 49-60.

Eberhard, S., Finazzi, G. \& Wollman, F. A. (2008). Annu. Rev. Genet. 42, 463-515.

Ehrmann, M. \& Clausen, T. (2004). Annu. Rev. Genet. 38, 709-724.

Eigenbrot, C., Ultsch, M., Lipari, M. T., Moran, P., Lin, S. J., Ganesan, R., Quan, C., Tom, J., Sandoval, W., van Lookeren Campagne, M. \& Kirchhofer, D. (2012). Structure, 20, 1040-1050.

Emsley, P., Lohkamp, B., Scott, W. G. \& Cowtan, K. (2010). Acta Cryst. D66, 486-501.

Fan, H., Sun, W., Sun, Z., Gao, F. \& Gong, W. (2012). Acta Cryst. F68, 839-841.

Haussühl, K., Andersson, B. \& Adamska, I. (2001). EMBO J. 20, 713-722.

Kapri-Pardes, E., Naveh, L. \& Adam, Z. (2007). Plant Cell, 19, 10391047.

Kato, Y., Sun, X., Zhang, L. \& Sakamoto, W. (2012). Plant Physiol. 159, 1428-1439.

Kim, S., Grant, R. A. \& Sauer, R. T. (2011). Cell, 145, 67-78.

Kim, D. Y., Kim, D. R., Ha, S. C., Lokanath, N. K., Lee, C. J., Hwang, H.-Y. \& Kim, K. K. (2003). J. Biol. Chem. 278, 6543-6551.

Kley, J., Schmidt, B., Boyanov, B., Stolt-Bergner, P. C., Kirk, R., Ehrmann, M., Knopf, R. R., Naveh, L., Adam, Z. \& Clausen, T. (2011). Nature Struct. Mol. Biol. 18, 728-731.

Komenda, J., Sobotka, R. \& Nixon, P. J. (2012). Curr. Opin. Plant Biol. 15, 245-251.

Krojer, T., Garrido-Franco, M., Huber, R., Ehrmann, M. \& Clausen, T. (2002). Nature (London), 416, 455-459.

Krojer, T., Sawa, J., Huber, R. \& Clausen, T. (2010). Nature Struct. Mol. Biol. 17, 844-852.

Krojer, T., Sawa, J., Schäfer, E., Saibil, H. R., Ehrmann, M. \& Clausen, T. (2008). Nature (London), 453, 885-890.

Kudla, J., Batistic, O. \& Hashimoto, K. (2010). Plant Cell, 22, 541-563.

Laskowski, R. A., MacArthur, M. W., Moss, D. S. \& Thornton, J. M. (1993). J. Appl. Cryst. 26, 283-291.

Li, W., Srinivasula, S. M., Chai, J., Li, P., Wu, J.-W., Zhang, Z., Alnemri, E. S. \& Shi, Y. (2002). Nature Struct. Biol. 9, 436-441.

Lohmiller, T., Cox, N., Su, J.-H., Messinger, J. \& Lubitz, W. (2012). J. Biol. Chem. 287, 24721-24733. 
Malet, H., Canellas, F., Sawa, J., Yan, J., Thalassinos, K., Ehrmann, M., Clausen, T. \& Saibil, H. R. (2012). Nature Struct. Mol. Biol. 19, 152-157.

Murshudov, G. N., Skubák, P., Lebedev, A. A., Pannu, N. S., Steiner, R. A., Nicholls, R. A., Winn, M. D., Long, F. \& Vagin, A. A. (2011). Acta Cryst. D67, 355-367.

Otwinowski, Z. \& Minor, W. (1997). Methods Enzymol. 276, 307326.

Rawlings, N. D., Morton, F. R., Kok, C. Y., Kong, J. \& Barrett, A. J. (2008). Nucleic Acids Res. 36, D320-D325.

Sakamoto, W. (2006). Annu. Rev. Plant Biol. 57, 599-621.

Schuhmann, H. \& Adamska, I. (2012). Physiol. Plant. 145, 224234.

Schuhmann, H., Huesgen, P. F. \& Adamska, I. (2012). BMC Plant Biol. 12, 52.

Shan, X., Sun, W., Fan, H., Jia, M., Gao, F. \& Gong, W. (2013). Acta Cryst. F69, 69-72.

Singh, N., Kuppili, R. R. \& Bose, K. (2011). Arch. Biochem. Biophys. 516, 85-96.

Sohn, J., Grant, R. A. \& Sauer, R. T. (2007). Cell, 131, 572583.
Stael, S., Wurzinger, B., Mair, A., Mehlmer, N., Vothknecht, U. C. \& Teige, M. (2012). J. Exp. Bot. 63, 1525-1542.

Sun, R., Fan, H., Gao, F., Lin, Y., Zhang, L., Gong, W. \& Liu, L. (2012). J. Biol. Chem. 287, 37564-37569.

Sun, X., Fu, T., Chen, N., Guo, J., Ma, J., Zou, M., Lu, C. \& Zhang, L. (2010). Plant Physiol. 152, 1263-1273.

Sun, X., Ouyang, M., Guo, J., Ma, J., Lu, C., Adam, Z. \& Zhang, L. (2010). Plant J. 62, 240-249.

Sun, X., Peng, L., Guo, J., Chi, W., Ma, J., Lu, C. \& Zhang, L. (2007). Plant Cell, 19, 1347-1361.

Truebestein, L., Tennstaedt, A., Mönig, T., Krojer, T., Canellas, F., Kaiser, M., Clausen, T. \& Ehrmann, M. (2011). Nature Struct. Mol. Biol. 18, 386-388.

Vagin, A. \& Teplyakov, A. (2010). Acta Cryst. D66, 22-25.

Voss, N. R. \& Gerstein, M. (2010). Nucleic Acids Res. 38, W555W562.

Wilken, C., Kitzing, K., Kurzbauer, R., Ehrmann, M. \& Clausen, T. (2004). Cell, 117, 483-494.

Winn, M. D. et al. (2011). Acta Cryst. D67, 235-242.

Wrase, R., Scott, H., Hilgenfeld, R. \& Hansen, G. (2011). Proc. Natl Acad. Sci. USA, 106, 13377-13382. 